\title{
Mathematical Principle Analysis of Transition Point Prediction
}

\author{
Peng YUE ${ }^{\mathrm{a}, \mathrm{b}, 1}$, Jinghui ZHANG ${ }^{\mathrm{a}}$ and Dewei PENG ${ }^{\mathrm{b}}$ and Sibei $\mathrm{WEI}^{\mathrm{c}}$ \\ ${ }^{a}$ University of Electronic Science and Technology of China, China \\ ${ }^{\mathrm{b}}$ China Aerodynamics Research and Development Center, China \\ ${ }^{\mathrm{c}}$ National Aerospace University, Kharkiv aviation institute, Ukraine
}

\begin{abstract}
Transition is the basic phenomena of complex flow regime changes in fluid mechanics. So far, the axiomatic expression and rigorous mathematical description of transition and separation are important basic scientific problems, which are also of great significance to the development of Applied Science. This article obtaining the basic conditions and general conditions for transition through the excited state theory of flowfield, which is derivation based on the N-S equations, combined with Euler's description point of view, applied tensor analysis and with the help of the basic principles of superposition states of quantum mechanics. The research of this paper provide a theoretical view for the study of turbulence mechanism.
\end{abstract}

Keywords. Fluid transition, Mathematical analysis, Transition point prediction, Fluid mechanics

\section{Introduction}

The flow state can usually be divided into laminar and turbulent. With the discovery of two flow regimes[1-2] and the concept of the boundary layer, the transition has become a major basic scientific problem that many scholars have been paying attention to for a long time and have not been solved. It characterizes the continuous change process of the system from a simple hierarchical stable state to a complex chaotic state. Today, although the vast number of scientific and technological workers in the field of fluid physics have invested a lot of manpower and material resources in research, they still have not found a universal theoretical method that can solve all turbulence problems. Since laminar and turbulent have a huge impact on frictional resistance, noise, fluidstructure coupling, and other characteristics[3], prediction accurately of the transition position not only has great engineering practical significance for the design of aircraft, engines, and underwater vehicles, but also very important academic value[4].

Experimental methods are effective means of research. Croci et al.[5] studied the laminar boundary layer separation. Chandra et al.[6] used particle image velocimetry to study the laminar turbulent transition. Miro et al.[7] quantified the effects of transition models on flow stability characteristics. Istvan et al.[8] studied the effect of free flow turbulence intensity. Wei et al.[9] studied the boundary layer transition. Although the

\footnotetext{
${ }^{1}$ Corresponding Author, Peng YUE, University of Electronic Science and Technology of China, China; Email: pengyu.yue@outlook.com.
} 
experiment can accurately show the physical process, due to the limitation of experimental conditions, the experimental results may have a certain deviation.

Because the numerical method can vividly display the calculation results, this method has attracted more and more attention from scholars. Sengupta et al.[10] solved the N-S equation to study the effects of forced frequency and free flow turbulence caused by unfavorable pressure gradients on the plate geometry. Salimipour[11] studied a numerical simulation of the transitional flow around different airfoils. Ni et al.[12] studied boundary-layer transition. Hosseinverdi et al.[13] carried out highly resolved DNS. Jiang et al.[14] used a DNS method to check the flow around a square cylinder for different Reynolds numbers.

In short, since the boundary layer theory was put forward, many scholars have carried out research on transition problems from different levels, improved the accuracy of numerical calculation methods, and made up for the lack of experimental simulation capabilities. According to Euler's description, the velocity can be regarded as the sum of the average velocity and the fluctuation velocity. Based on the theroy of superposition state, the general law of transition is obtained, and the theoretical results are consistent with the experimental results.

\section{Mathematical analysis of Transition}

According to the N-S equations, the velocity field $u_{i}$ satisfies:

$$
\begin{gathered}
\frac{\partial \rho}{\partial t}+\left(\rho u_{i}^{\cdot}\right)^{, i}=0 \\
\rho \frac{\partial u_{i}}{\partial t}+\rho u_{j} u_{i, \cdot}^{\cdot j}=\sigma_{i j, \bullet}^{\cdot j}+f_{i}
\end{gathered}
$$

where $\rho$ is the density, and $f_{i}$ is the body force. $\sigma_{i j}$ is the stress state tensor, which is:

$$
\sigma_{i j}=-p \delta_{i j}+\psi \mu u_{k, \cdot}^{\cdot, k} \delta_{i j}+\mu\left(u_{j, i}+u_{i, j}\right)
$$

here $p$ is the pressure, $\delta_{i j}$ is the Kronecker symbol, and $\mu$ is coefficient of dynamic viscosity.

According to the Reynolds equation[15-16], the velocity[17] can express as (Figure 1):

$$
u_{i}=u_{i}^{\prime}+\bar{u}_{i}
$$

where $u_{i}^{\prime}$ is the fluctuation velocity and $\bar{u}_{i}$ is the average velocity.
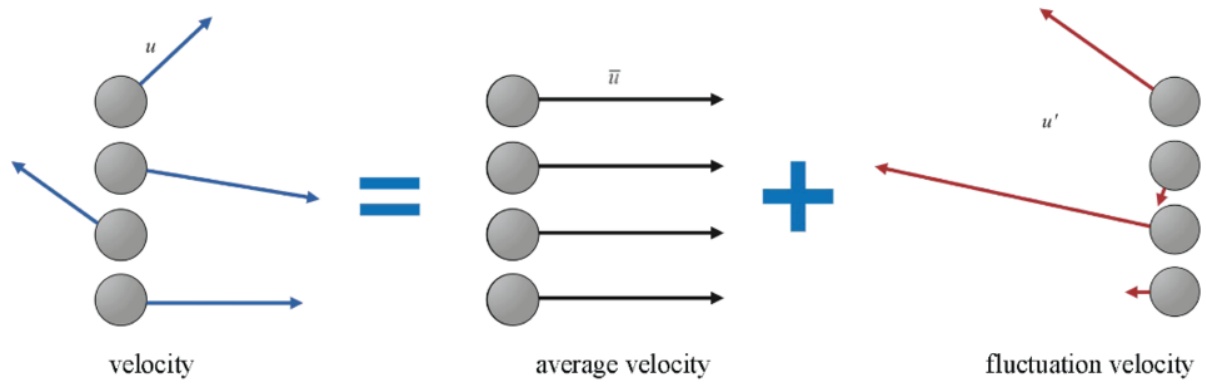

Figure 1. Schematic diagram of velocity. 
The features of transition[18] are randomness and irregularity. Let $U\left(x_{h}, \varepsilon\right)$ be a neighborhood of point $x_{h}$. A laminar fluid with $u_{i}$ flows through the neighborhood, and excites $u_{j}^{\prime}$. Let $\forall x_{k} \in U\left(x_{h}, \varepsilon\right), \exists x_{s} \in U\left(x_{h}, \varepsilon\right)$ and $x_{s} \neq x_{k}$. If they satisfy:

$$
u_{i}\left(x_{k}\right)=u_{i}\left(x_{s}\right), u_{j}^{\prime}\left(x_{k}\right) \neq u_{j}^{\prime}\left(x_{s}\right), u_{j}^{\prime}\left(x_{h}\right)=0
$$

then the point $x_{h}$ is called the transition point.

For $x_{j} \in\left\{x_{i}\left|x_{i} \in U\left(x_{k}, \varepsilon\right),\right| x_{k}-x_{i} \mid<\varepsilon, \forall \varepsilon>0\right\}$, the conditions of generating $u_{i}^{\prime}$ is:

$$
u_{j}^{\prime}=0
$$

so:

$$
\frac{d u_{j}^{\prime}}{d t}=\frac{\partial u_{j}^{\prime}}{\partial t}+u_{k} u_{j, \bullet}^{\prime \bullet, k} \neq 0
$$

as $\varepsilon \rightarrow 0, x_{k}$ gives the coordinates of the transition points.

According to the concept of the superposition state[19] (Figure 2):

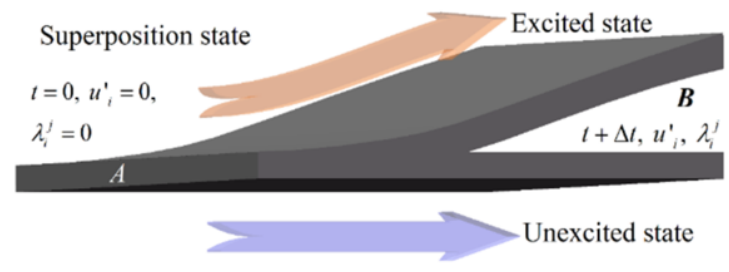

Figure 2. Superposition state.

Excited state is that the fluctuation velocity has been generated. We set up a secondorder tensor satisfies:

$$
u_{i}^{\prime}=\bar{u}_{j} \lambda_{i}^{j}, u_{i}^{\prime}, \bar{u}_{j} \in \mathcal{J}_{M}
$$

then $\lambda_{i}^{j}$ is called the velocity strain tensor.

From equation (6) and (7), $\lambda_{i}^{j}$ satisfies:

$$
\lambda_{i}^{j}=0
$$

the time derivative of $\lambda_{i}^{j}$ is:

$$
\frac{\mathrm{d} \lambda_{i}^{j}}{\mathrm{~d} t}=\frac{\partial \lambda_{i}^{j}}{\partial t}+u_{k} \lambda_{i, \bullet}^{j, k} \neq 0
$$

where $u_{k}$ is the velocity field of the flow.

In the unexcited state, $u_{i}^{\prime}=0$. By equation (1) and (2), the unexcited state equations are:

$$
\frac{\partial \rho}{\partial t}+\rho^{\bullet, i} \bar{u}_{j} \delta_{i}^{j}+\rho \bar{u}_{j, \cdot}^{\cdot, i} \delta_{i}^{j}=0
$$




$$
\rho \frac{\partial\left(\bar{u}_{j} \delta_{i}^{j}\right)}{\partial t}+\rho \bar{u}_{j 1} \bar{u}_{j 2,}^{\cdot k}, \delta_{k}^{j 1} \delta_{i}^{j 2}=g_{i}^{(1)}
$$

where $g_{i}^{(1)}=\sigma_{i k,}^{(1) \cdot, k}+f_{i}$ is the external force field in unexcited state, $\sigma_{i k}^{(1)}$ is the stress state tensor in unexcited state, and $\delta_{i j}$ is unit constant tensor.

In the excited state, introducing the excited ratio tensor:

$$
\xi_{i}^{j}=\delta_{i}^{j}+\lambda_{i}^{j}
$$

The following conclusion is drawn.

$$
u_{i}=\bar{u}_{j} \delta_{i}^{j}+\bar{u}_{j} \lambda_{i}^{j}=\bar{u}_{j}\left(\delta_{i}^{j}+\lambda_{i}^{j}\right)=\bar{u}_{j} \xi_{i}^{j}
$$

For the excited state, $\xi_{i}^{j}$ satisfies:

$$
\begin{gathered}
\frac{\partial \rho}{\partial t}+\rho^{\cdot, i} \bar{u}_{j} \xi_{i}^{j}+\rho \bar{u}_{j, \cdot}^{, i} \xi_{i}^{j}+\rho \bar{u}_{j} \xi_{i, \cdot}^{j, i}=0 \\
\rho \frac{\partial\left(\bar{u}_{j} \xi_{i}^{j}\right)}{\partial t}+\rho \bar{u}_{j 1} \bar{u}_{j 2, \cdot, \cdot} e_{k i}^{j 1 j 2}+\rho \bar{u}_{j 1} \bar{u}_{j 2} \alpha_{i}^{j 1 j 2}=g_{i}^{(2)}
\end{gathered}
$$

where $g_{i}^{(2)}=\sigma_{i k,}^{(2) \cdot, k}+f_{i}$ is the external force field in excited state, $\sigma_{i k}^{(2)}$ is the stress state tensor, $e_{k i}^{j 1 j 2}=\xi_{k}^{j 1} \xi_{i}^{j 2}$, and $\alpha_{i}^{j 1 j 2}=\xi_{k}^{j 1} \xi_{i,}^{j 2, k}$ are the adjustment tensor coefficients.

For the excited process, the $\lambda_{i}^{j}$ satisfies:

$$
\begin{gathered}
\rho^{\cdot, i} \bar{u}_{j} \lambda_{i}^{j}+\rho \bar{u}_{j, \cdot}^{\cdot,} \lambda_{i}^{j}+\rho \bar{u}_{j} \lambda_{i, \cdot}^{j, i}=0 \\
\rho \frac{\partial\left(\bar{u}_{j} \lambda_{i}^{j}\right)}{\partial t}+\rho \bar{u}_{j 1}\left(\bar{u}_{j 2, \cdot}^{\cdot, k} \varepsilon_{k i}^{j 1 j 2}+\bar{u}_{j 2} \xi_{k}^{j 1} \lambda_{i, \bullet}^{j 2, k}\right)=g_{i}
\end{gathered}
$$

where $g_{i}=g_{i}^{(2)}-g_{i}^{(1)}=\sigma_{i k, \cdot(2) \cdot, k}-\sigma_{i k, \cdot}^{(1) \cdot, k}$, The adjustment tensor coefficient $\varepsilon_{k i}^{j 1 j 2}=e_{k i}^{j 1 j 2}-\delta_{k}^{j 1} \delta_{k}^{j 2}=\lambda_{k}^{j 1} \lambda_{i}^{j 2}+\lambda_{k}^{j 1} \delta_{k}^{j 2}+\delta_{k}^{j 1} \lambda_{i}^{j 2}$.

Equation (11) and (12) are substituted into equation (17) and (18), we can get:

$$
\begin{gathered}
\lambda_{i, \cdot}^{j, i}=0 \\
\rho \bar{u}_{j} \frac{\partial \lambda_{i}^{j}}{\partial t}+\rho \bar{u}_{k} \bar{u}_{j} \lambda_{i, \cdot}^{j, k}=g_{i}
\end{gathered}
$$

For the unexcited state, the gradient of the stress tensor of the flowfield could be expressed as:

$$
\sigma_{i k, \cdot}^{(1) \cdot \bullet, k}=-p_{(1)}^{\bullet, k} \delta_{i k}+\psi \mu \bar{u}_{t, . \bullet}^{\bullet, s k} \delta_{s}^{t} \delta_{i k}+\mu\left(\bar{u}_{j, k \cdot}^{\cdot \bullet \cdot} \delta_{i}^{j}+\bar{u}_{j, i \bullet}^{\cdot \bullet k} \delta_{k}^{j}\right)
$$

For the excited state, the gradient of the stress tensor of the flowfield could be expressed as:

$$
\begin{aligned}
& \sigma_{i k, \bullet}^{(2) \cdot, k}=-p_{(2)}^{\cdot, k} \delta_{i k}+\psi \mu \bar{u}_{t, *}^{\cdot, k} \xi_{s}^{t} \delta_{i k}+\psi \mu \bar{u}_{t, \cdot}^{\cdot, s} \xi_{s, \bullet}^{t, k} \delta_{i k}+\psi \mu \bar{u}_{t, \cdot}^{\cdot, k} \xi_{s, \bullet}^{t, s} \delta_{i k} \\
& +\psi \mu \bar{u}_{t} \xi_{s, \bullet}^{t, s k} \delta_{i k}+\mu \bar{u}_{j, k \cdot}^{\cdot \bullet \cdot} \xi_{i}^{j}+\mu \bar{u}_{j, k} \xi_{i, \bullet}^{j, k}+\mu \bar{u}_{j, \cdot}^{\cdot, k} \xi_{i, k}^{j, \cdot}++\mu \bar{u}_{j} \xi_{i, k \cdot}^{j, \bullet k} \\
& +\mu \bar{u}_{j, i}^{\bullet \cdot k} \xi_{k}^{j}+\mu \bar{u}_{j, i} \xi_{k, \bullet}^{j, k}+\mu \bar{u}_{j, \cdot, k} \xi_{k, i}^{j, \bullet}+\mu \bar{u}_{j} \xi_{k, i \bullet}^{j, \cdot k}
\end{aligned}
$$

The stress difference between two states is: 


$$
\begin{aligned}
& g_{i}=\sigma_{i k, \bullet}^{(2) \bullet, k}-\sigma_{i k, \bullet}^{(1) \bullet, k}=p_{\bullet, i}^{(1)}-p_{\bullet, i}^{(2)}+\mu \bar{u}_{j, k} \lambda_{i, \bullet}^{j, k}+\psi \mu\left(\bar{u}_{j, \bullet}^{\bullet, k} \lambda_{k, i}^{j, \bullet}+\bar{u}_{j} \lambda_{k, i \bullet}^{j, \bullet}\right) \\
& +\mu\left(\bar{u}_{j, \cdot}^{\cdot, k} \lambda_{i, k}^{j, \bullet}+\bar{u}_{j} \lambda_{i, k \cdot \bullet}^{j, \bullet}\right)+\mu\left(\bar{u}_{j, \cdot}^{\bullet, k} \lambda_{k, i}^{j, \bullet}+\bar{u}_{j} \lambda_{k, i \cdot}^{j, \cdot}\right)
\end{aligned}
$$

The final excitation law is:

$$
\begin{gathered}
\lambda_{i}^{j}=0, \lambda_{i, \bullet}^{j, i}=0 \\
\rho u_{j} \frac{\partial \lambda_{i}^{j}}{\partial t}+\rho u_{k} u_{j} \lambda_{i, \bullet}^{j, k}=P_{i}+\mu u_{j, k} \lambda_{i, \bullet}^{j, k}+\mu\left(u_{j} \lambda_{i, k}^{j, \bullet}+\phi u_{j} \lambda_{i, k}^{j, \bullet}\right)^{, k}
\end{gathered}
$$

where $\phi=\psi+1$.

We introduce the degenerate condition[20] to solve above equations. There is:

$$
\frac{d x_{k}}{d t}=\frac{\partial x_{k}}{\partial t}
$$

According to the difference principle, $u_{i}^{\prime}$ meets:

$$
\rho \frac{\partial u_{i}^{\prime}}{\partial t}=\frac{P_{i}+P_{i}^{\prime}}{1+\gamma}
$$

where $\gamma=1-\beta ; P_{i}$ is difference of the pressure gradient. Let the fluctuation velocity gradient is $C_{k i}^{\prime}$, the characteristic pressure $P_{i}^{\prime}$ is:

$$
P_{i}^{\prime}=\mu\left(\phi \frac{\partial C_{k i}^{\prime}}{\partial t}+\frac{\partial C_{i k}^{\prime}}{\partial t}\right)\left(u_{k}\right)^{-1}
$$

let velocity gradient is $C_{k i}$, the viscosity coefficient $\beta=\mu C_{k j}\left(\rho u_{k} u_{j}\right)^{-1}$, where $\mu C_{k j}$ is the viscous stress.

The pressure gradient difference is:

$$
P_{i}=p_{\bullet, i}^{(1)}-p_{\bullet, i}^{(2)}=-\Delta p_{\bullet, i}
$$

where $\Delta p_{, i}$ is the variation of the pressure gradient.

Let $\zeta$ evaluate the spatial variation rate, and $\Delta x_{i}$ represents the scale of spatial change. There is:

$$
\xi=\lim _{\Delta x_{i} \rightarrow 0} \frac{\Delta p_{\cdot, i}}{\Delta x_{i}}=\mathcal{L} p
$$

where $\mathcal{L}$ is the Laplace operator. At the same time, we have:

$$
\frac{\partial \theta^{\prime}}{\partial t}=\frac{1}{1+\gamma}\left(\frac{P_{i, \bullet}^{\prime \bullet i}}{\rho}-\frac{\mathcal{L} p}{\rho}\right)
$$

where $\theta^{\prime}=u_{j, \bullet}^{\prime \bullet, j}$ is fluctuation velocity expansion.

That if $u_{k}=0$, then $P_{i, \cdot}^{\prime \cdot,}=0$; if $u_{k} \neq 0$, then:

$$
P_{i, \bullet}^{\prime, \cdot}=\frac{n v}{n+1}\left[(1+\phi) p_{\bullet, k}^{\prime} v^{k}-\eta p^{\prime}\left(u_{i} v_{k}+\phi u_{k} v_{i}\right) C^{k i}\right]+\frac{v \eta \theta}{(n+1)^{2}}\left[(1+\phi) n p^{\prime}-\left(f_{i}^{\prime} v_{k}+\phi f_{k}^{\prime} v_{i}\right) C^{k i}\right]
$$

where $n$ is the spatial dimension. 
Transition represents the generation of irregular pulsation speed, and its initial value is very small. Therefore, $u_{i}^{\prime} \rightarrow 0, f_{i}^{\prime} \rightarrow 0, p^{\prime} \rightarrow 0$, and $p_{\cdot, k}^{\prime} \rightarrow 0$, it could be considered that $P_{i, \bullet}^{\prime \bullet, i} \rightarrow 0$.

Let $U\left(x_{h}, \varepsilon\right)$ be a neighborhood of point $x_{h}$, then:

$$
\mathcal{L} p\left(x_{h}\right) \neq 0, \mathcal{L} p\left(x_{k}\right)=0, \forall x_{k} \in U\left(x_{h}, \varepsilon\right)
$$

all points $x_{j}$ satisfying the above equation, which denotes the transition position.

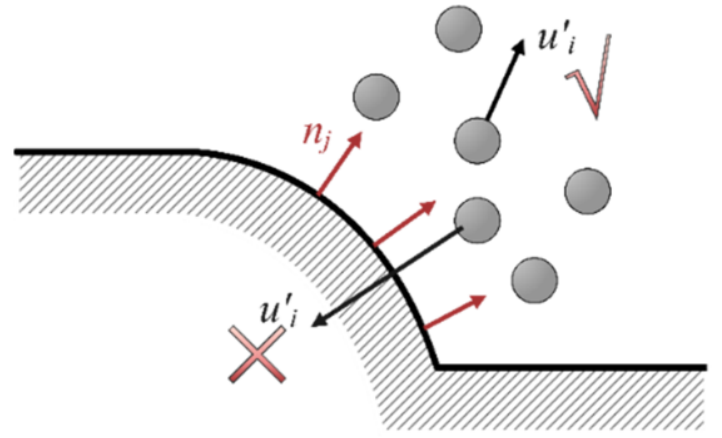

Figure 3. Wall condition.

According to equation (30), we could get:

If $n^{i}>0$, then $\mathcal{L} p-P_{i, \bullet}^{\prime \bullet, i}<0$; conversely, then $\mathcal{L} p-P_{i, \bullet}^{\prime \bullet, i} \geq 0$

where $n^{i}$ is the outer normal vector of the solid boundary(figure 3 ).

If the system is excited, there is a scalar physical variable $\Pi$ that satisfies:

$$
\Pi=\frac{1}{1+\gamma}\left(\frac{\mathcal{L} p}{\rho}-\frac{P_{i, \cdot}^{\prime \cdot i}}{\rho}\right)
$$

Now we find that:

$$
\frac{d^{2} C_{p}}{d x^{2}}=0
$$

where $C_{p}$ is the pressure coefficient, $x$ is the transition positions, and $\varepsilon \rightarrow 0$ is infinitesimal, .

Due to the above conditions, the transition point should meet:

If $n^{i}>0$, then $\frac{d^{2} C_{p}}{d x^{2}}(x-\varepsilon)<0$; conversely, then $\frac{d^{2} C_{p}}{d x^{2}}(x-\varepsilon) \geq 0$

\section{Experimental Validation}

In the one-dimensional flow experiment, the airfoil NACA2412 with an inflow velocity of $30 \mathrm{~m} / \mathrm{s}$ was employed, and $\alpha=-2^{\circ}$. The experimental device is shown in figure 4 , and the pressure curve is shown in figure 5. 


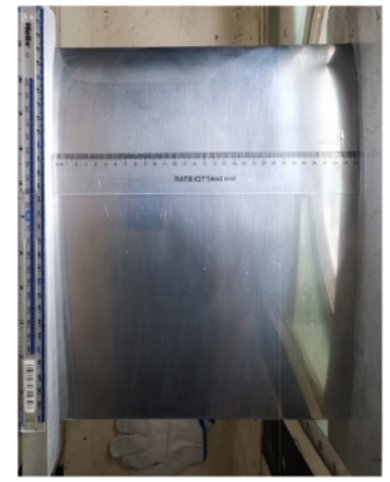

Figure 4. Experimental device.

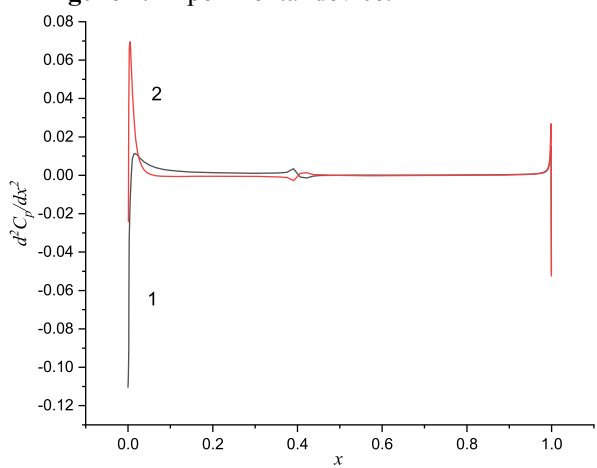

Figure 6. Second derivative curve.

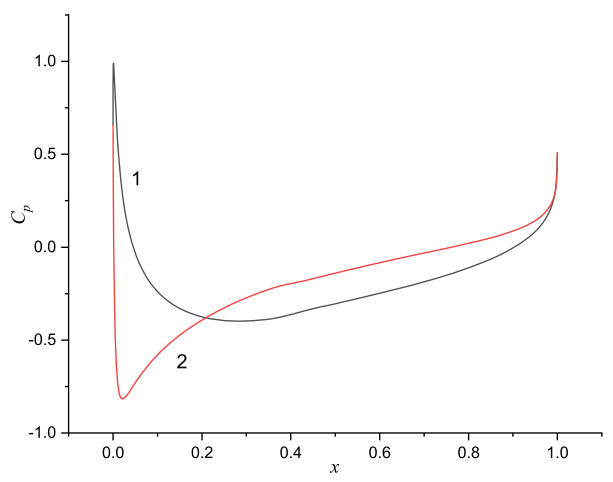

Figure 5. Pressure curve.

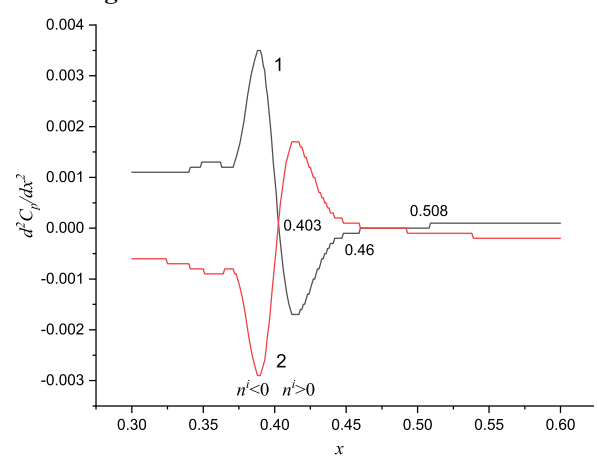

Figure 7. The second derivative curve of pressure.

The derivative curves are shown in the figure 6 and figure 7. According to the results in figure 6 and figure 7, the predicted value is $x=0.403$ with transition and $x=0.508$ with full transition.

The experimental results are shown in figure 8 . The value is $x=0.405$ with initial transition and $x=0.502$ with full transition.

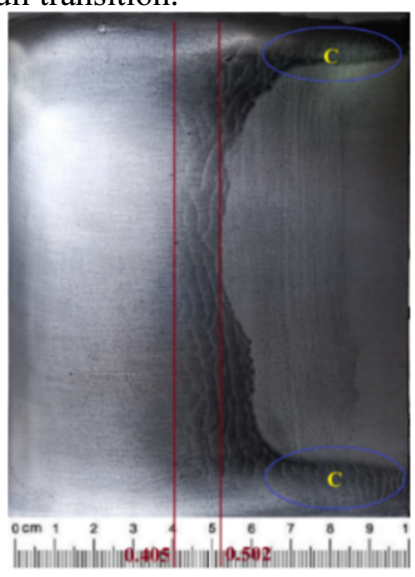

Figure 8. Experiment result.

The prediction accuracy is: 


$$
\Delta_{1}=\frac{|0.403-0.405|}{0.405}=0.49 \% \quad \Delta_{2}=\frac{|0.508-0.502|}{0.404}=1.19 \%
$$

According to the above calculation results, both errors are less than $2 \%$.

Many literature studies show that it is possible to predict the transition position according to the measurement of surface pressure distribution. Popov et al[21] found a method to determine the transition position according to the pressure distribution, and the transition point is the maximum curvature in the pressure distribution, and the result is shown in figure 9. Boutilier et al[22] measured the pressure distribution of NACA0018 airfoil from $5 \times 10^{4}$ to $2.5 \times 10^{5}$ Reynolds numbers through experiments, and studied the transition characteristics on the airfoil surface, they found that the transition, separation and reattachment occurred near the pressure plateau, the phenomenon is shown in figure 10.
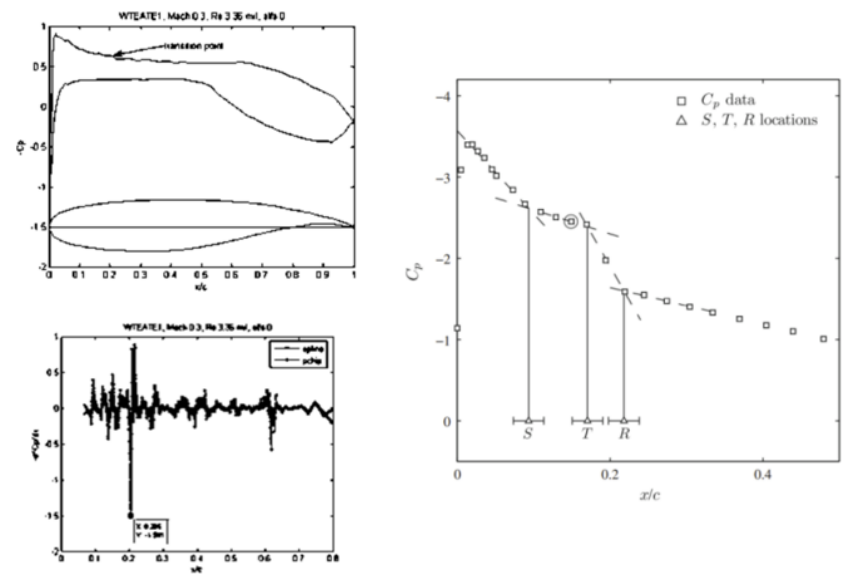

Figure 9. $C_{p}$ distributions of the WTEA-TE1 airfoil and the second derivative of $C_{p}$.

Figure 10. Surface pressure data $(R e=$ 150,000 and $\alpha=10^{\circ}$ ).

\section{Conclusion}

In order to determine the transition positions, this paper uses the superposition state, and modern applied mathematics methods to propose the fluid excited state method. At the same time, this paper puts forward the principles of mechanics, obtains the basic tensor equations of excited states, and proposes the concept of degenerate form to establish the law of transition. In addition, this paper obtains the excitation degeneracy through discrete time and space and put forward the basic theory of transition.

\section{Reference}

[1] Hall M 1981 Computational fluid dynamics-A revolutionary force in aerodynamics 5th Computational Fluid Dynamics Conference p 1014

[2] Viviand H, Lecomte C and Morice P 1987 Computational fluid dynamics in France 8th Computational Fluid Dynamics Conference p 1131 
[3] Balzer W and Fasel H F 2016 Numerical investigation of the role of free-stream turbulence in boundarylayer separation Journal of Fluid Mechanics 801 289-321.

[4] Yang Z 2019 On bypass transition in separation bubbles: a review Propulsion and Power Research 8(1) 23-34

[5] Croci K, Ravelet F, Danlos A, Robinet J C and Barast L 2019 Attached cavitation in laminar separations within a transition to unsteadiness Physics of Fluids 31(6) 063605

[6] Chandra B, Shankar V and Das D 2019 Onset of transition in the flow of polymer solutions through deformable tubes Physics of Fluids 31(11) 114103

[7] Miró Miró F, Beyak E S, Pinna F and Reed H L 2019 High-enthalpy models for boundary-layer stability and transition Physics of Fluids 31(4) 044101

[8] Istvan M S, Kurelek J W and Yarusevych S 2018 Turbulence intensity effects on laminar separation bubbles formed over an airfoil AIAA Journal 56(4) 1335-1347

[9] Wei B, Gao Y, Wang L and Li D 2019 Analysis of flow transition and separation on oscillating airfoil by pressure signature Journal of Mechanical Science and Technology 33(1) 279-288

[10] Sengupta A and Tucker P 2020 Effects of forced frequency oscillations and free stream turbulence on the separation-induced transition in pressure gradient dominated flows Physics of Fluids 32(10) 104105

[11] Salimipour E 2019 A modification of the k-kL- $\omega$ turbulence model for simulation of short and long separation bubbles Computers \& Fluids 181 67-76

[12] Ni W, Lu L, Fang J, Moulinec C, Emerson D R and Yao, Y 2019 Flow separation control over a rounded ramp with spanwise alternating wall actuation Physics of Fluids 31(1) 015101

[13] Hosseinverdi S and Fasel H F 2018 Role of Klebanoff modes in active flow control of separation: direct numerical simulations Journal of Fluid Mechanics 850 954-983

[14] Jiang H 2020 Separation angle for flow past a circular cylinder in the subcritical regime Physics of Fluids 32(1) 014106

[15] Du Z B, Huang W, Yan L and Li S B 2019 Reynolds-average Navier-Stokes study of steady and pulsed gaseous jets with different periods for the shock-induced combustion ramjet engine Physics of Fluids 31(5) 055107

[16] Graham M D and Floryan D 2021 Exact coherent states and the nonlinear dynamics of wall-bounded turbulent flows Annual Review of Fluid Mechanics 53 227-253

[17] Yan F, Rinoshika A, Tang W and Zhu R 2019 Experimental analysis on particle fluctuation velocity in a horizontal air-solid two-phase pipe flow having a dune model Particulate Science and Technology 37(2) 182-189

[18] Majumdar D, Bose C and Sarkar S 2020 Capturing the dynamical transitions in the flow-field of a flapping foil using immersed boundary method Journal of Fluids and Structures 95102999

[19] Chiribella G 2020 Quantum superpositions of causal structures Critical Hermeneutics 4(special II) 1-24

[20] Zhan H and Feng Z 2021 Optimal partial boundary condition for degenerate parabolic equations Journal of Differential Equations 284 156-182

[21] Popov A V, Botez R M and Labib M 2008 Transition point detection from the surface pressure distribution for controller design Journal of Aircraft 45(1) 23-28

[22] Boutilier M S and Yarusevych S 2012 Parametric study of separation and transition characteristics over an airfoil at low Reynolds numbers Experiments in fluids 52(6) 1491-1506 